assumed that two provocatives of morbid action-in short, two viruses-cannot work at once in the same place. But the syphilitic poison is not so antagonistic to other agents of diseased action, for it has been proved that it may be transferred with the vaccine lymph from one child to another, and there its action and development are not prevented by the presence and previous formation of the vaccine vesicle and cicatrix. Syphilis in these cases-for a particular account of which I must refer my readers to M. Viennois's (of Lyons) treatise on the Propagation of Syphilis by Vaccination-has a course quite similar to those where it is engrafted without vaccinia.

In conclusion, I may be allowed to enumerate the points I have endeavoured to establish :-

1. That the evolution of primary syphilis is generally, but not invariably, accompanied by ulceration.

2. This ulceration is not the earliest sign of successful inoculation, but rather a result of degeneration of the badly developed tissue of which the indurated papule or tubercle consists.

3. That the production of an ulcer immediately after inoculation is in every case due, not to the syphilitic poison itself, but to irritating matter being inserted with it in the wound; this irritating matter being in many cases the contagious principle of soft chancres, or the pus of other suppurating surfaces, as'in the instance mentioned before, where Vidal imserted some pus of a syphilitic pustule into a gentleman's hand, and produced a pustule which lasted fifteen days, then subsided, and no further action took place until the thirty-fifth day, when the eruption broke out afresh, and constitutional symptoms appeared 128 days after the inoculation.

4. That the ordinary commencement of syphilis being by formation of a solid elevation of the skin at the point of entry of the poison, with subsequent ulceration of the tubercle, it is very doubtful if these appearances are ever altogether absent.

Weymouth-street, Oct. 1865.

\section{ON PROLAPSUS OF THE RECTUM, WITH H.AMORRHOIDS.}

ABSCESS AND FISTULOUS COMMUNICATION WITH THE BOWEL.

By DAVID JOHNSON, M.D., M.R.C.S.

TuE following is one of those cases which, while rendering the patient's life one of constant misery and pain, is too frequently, from motives of delicacy, obscured from the pale of the surgeon's interference, although a speedy and permanent cure could in perhaps all instances be obtained :-

H. S- - aged forty-three, a clerk, of sedentary habits, consulted me for disease of the rectum. He is a dark, emaciated, but well-proportioned gentleman, with an exceedingly sallow and jaundice-looking complexion. He formerly resided in Calcutta, and was removed thither to London eighteen months since in consequence of his present indisposition. He was invariably constipated, and had for years been the subject of a prolapsed. condition of the bowel associated with piles. Defecation was always followed by throbbing and smarting pain in the fundament, accompanied with violent straining and oozing of blood. Micturition, too, was attended with great pain and bearingdown, but there was no urinary disease. While in India he was attended for occasional hepatic mischief, and felt sure he had taken more than his share of blue-pill and aloes, as he had lost several of his teeth from excessive ptyalism. Various kinds of unguents and injections had been had recourse to locally without any appreciable beneficial result. He had now no appetite, was very restless and miserable, rarely being able to sleep without resorting to opium. He seemed desponding, and evinced little desire to change his position-in short, his ailment had grown so troublesome that he shunned society, and was confined to his bedroom. If he attempted to raise himself to the erect posture, the prolapsus was instantaneous and complete, causing pain of the most agonizing and intolerable kind, and while in that position blood would trickle down to his feet. It was in this deplorable condition he sought my advice.

On examination, I found a large globular mass of mucous membrane and intestine protruding, forming a tumour the size of my fist, exceedingly vascular, and covered with blood, and so exquisitely sensitive that he dreaded my attempting to touch it. On the left margin of the anus there was a large hxmorrhoid ; while on the right, and somewhat anteriorly, a fluctuating abscess presented itself, the size of a walnut. 'This I immediately opened, and gave exit to a quantity of pus and dark-coloured ituid, of a most horribly offensive character not easily to be forgotten. By gentle pressure with a warm sponge I readily succeeded in returning the protrusion; still a slight cough or strain quickly brought the mass into view again, evidently due to the remarkably relaxed condition of the sphincter ani muscle, caused by its constant descent. By making now a digital examination with the left index-finger high up in the rectum, and at the same time passing a director through the abscess wound, a fistulous communication with the bowel was found to exist. While manipulating, a small, hard, and round body, not unlike a cherry-stone, escaped through the abscess wound, and which had doubtless made its way through the mncons membrane, and, burrowing there, had given rise to this auditional trouble.

After desiring a dose of castor oil to be taken at night, I visited him the following morning, and, finding the protrusion complete, cautiously but freely applied strong nitric acid over the entire surface, which brought on a rood deal of involuntary straining, and, on its subsiding, I returned the substance within the sphincter, and administered two grains of solid opium. This occurred on the 15th of January last. On the 22nd, I had applied the acid every other day, having aided the descent of the bowel; and there was now a good deal of apparent sloughing, but less protrusion. Bowels open every other day, and he did not complain of so much pain.

Jan. 29th.-Dressed with the acid twice; bowels confined; appetite bad; in great pain, and complains of tenesmus. Adniinistered an enema of cold water, with glycerine and laudanum, prescribed the following mixture and pills, and recom. mended a soft but nutritious diet, with a moderate quantity of wine :-Dilute hydrochloric acid, one drachm and a half; tincture of calumba, one oince; water to six ounces : an ounce to be taken thrice a day. Extract of nux vomica, two grains extract of hyoscyamus, twelve grains; a sufficient quantity of conserve of roses to make six pills : one to be taken every night.

Feb. 4th.-Health much improved; very cheerful; appetite good; bowels obedient; no protrusion whatever; no pain; a little discharge of blood and pus, which is increased after defecation.

5th.-I divided the sphincter, and laid bare the fistula; dressed with dry lint, and ordered occasional ablution with soap-and-water; inserted an opium suppository. To discontinue the nux-vomica pills. Absolute quietude enjoined.

10th.-Improving; passed is double ligature through the base of the external hwmorrhoid; bowels confined. The pills to be recommenced. Appetite very good; wounds healing.

17th.-Still progressing; ligatures separated; wounds nearly healed; is very anxious to get up.

26th.-Walking about the house. Expresses himself well and able to resume employment. Defecates daily without inconvenience or pain.

Since these notes were taken I have frequently met him walking to and from his occupation, which he now says is his luxury. He has gained considerably in flesh, and, in his own words, "is as happy as a king."

Old Kent-rosd, Oet. 1865.

Proposed New Professofiship at Cambridge.The Syndicate appointed to consider the best mode of providing for the teaching of anatomy and zoology in the university has made a report to the Senate, which has already been printed. After stating that at Lady-day, 1866, a charge amounting to $£ 300$ a year, for a portion of the stipends of the Lucasian and Plumian Professors, will cease, the Syndicate recommended-1. That the stipend of $£ 300$ per annum now paid to the Professor of Anatomy be continued to his immediate successor, who may be expected to take the department of Human Anatomy and Physiology. 2. That a Demonstrator in Anatomy be appointed at a stipend of $£ 100$ per annum. 3. That a Professor of Zoology and Comparative Anatomy be appointed at a stipend of $£ 300$ per annum, to lecture on, and teach the principles of these sciences. A meeting, convened by the Vice-Chancellor, to consider the terms of this report was held in the Arts School on Saturday afternoon, the Vice-Chancellor presiding. Several scientific men present discussed the question, and the general tone of the meeting was favourable to the proposed changes. 